\title{
Metabolic efficiency with fast spiking in the squid axon
}

\section{Abdelmalik Moujahid* and Alicia d'Anjou}

Computational Intelligence Group, Department of Computer Science, University of the Basque Country UPVIEHU, San Sebastián, Spain

\section{Edited by:}

Julie Wall, Queen Mary, University of London, UK

\section{Reviewed by:}

Julie Wall, Queen Mary, University

of London, UK

Christoph Borgers, Tufts University, USA

\section{*Correspondence:}

Abdelmalik Moujahid

Computational Intelligence Group,

Department of Computer Science,

University of the Basque Country

UPV/EHU, 20018 San Sebastián,

Spain.

e-mail: jibmomoa@gmail.com
Fundamentally, action potentials in the squid axon are consequence of the entrance of sodium ions during the depolarization of the rising phase of the spike mediated by the outflow of potassium ions during the hyperpolarization of the falling phase. Perfect metabolic efficiency with a minimum charge needed for the change in voltage during the action potential would confine sodium entry to the rising phase and potassium efflux to the falling phase. However, because sodium channels remain open to a significant extent during the falling phase, a certain overlap of inward and outward currents is observed. In this work we investigate the impact of ion overlap on the number of the adenosine triphosphate (ATP) molecules and energy cost required per action potential as a function of the temperature in a Hodgkin-Huxley model. Based on a recent approach to computing the energy cost of neuronal action potential generation not based on ion counting, we show that increased firing frequencies induced by higher temperatures imply more efficient use of sodium entry, and then a decrease in the metabolic energy cost required to restore the concentration gradients after an action potential. Also, we determine values of sodium conductance at which the hydrolysis efficiency presents a clear minimum.

Keywords: Hodgkin-Huxley model, action potential, neuron metabolic energy, sodium entry, overlap load, regular-spiking cells

\section{INTRODUCTION}

The generation of action potentials in mammalian neurons involves the flux of different ions such as sodium, potassium, and calcium across the cell membrane. In this process, the electrochemical gradients are partially altered and must be restored by ion pumps which move ions from one side of the membrane to the other at the expense of energy. Re-establishing the concentration gradients after electrical discharges demands most of the energy used for neuronal metabolism (Laughlin et al., 1998; Laughlin, 2001; Shulman et al., 2004; Siekevitz, 2004). This requirement for metabolic energy has important implications for the brain's evolution and function (Attwell and Laughlin, 2001), and the availability of energy may impose a limit on neural activity taking into account that the brain has very small energy reserves (Ames III, 2000). It is, however, generally accepted that energy metabolism is highly organized within cells resulting in energetically efficient mechanisms that transfer energy from the site of generation to the processes that require it (Ames III, 2000; Belanger et al., 2011).

On other hand, all energy used for neural metabolism is finally transformed into heat (Ames III, 2000), and the metabolic brain activation appears to be the primary cause of heat production. Because neural properties are temperature-dependent, potential imbalance between heat production and dissipation could lead to overheating and aberrant functioning (Falk, 1990; Koch, 1998; Kiyatkin, 2007). Studying the relationship between temperature, firing frequency, sodium entry, and the energy cost required to generate an action potential using neuron models like the Hodgkin-Huxley model (Hodgkin, 1975) will provides a useful framework for addressing these issues.
The Hodgkin-Huxley model representing the dynamics of the squid giant axon continues to be the most frequently used model to study the dynamics and other properties of actual neurons. Based on biophysical considerations about the nature of the Hodgkin-Huxley model, we have recently found an analytical expression of the electrochemical energy involved in the dynamics of the model, which provides a new approach for estimating the energy consumption during the resting and active states of neurons (Moujahid et al., 2011). This energy function was used as a measure to evaluate the metabolic energy consumption of a neuron to maintain its signaling activity and to estimate the metabolic cost of transmitting information between neurons.

This approach, contrary to other methods (Attwell and Laughlin, 2001; Lennie, 2003), does not require ion counting for estimating the metabolic energy consumption of the generation of action potentials, and gives us the opportunity to check in the Hodgkin-Huxley model which ion counting gives the correct metabolic energy consumption. In this work we investigate the impact of ion currents overlapping on the number of adenosine triphosphate (ATP) molecules required to restore the concentration gradients after an action potential in the Hodgkin-Huxley model. Because the observed overlap is temperature-dependent, we have computed the number of ATP molecules per action potential and its corresponding energy cost at different values of temperature. Both the classic study by Hodgkin and Huxley of the squid axon (Hodgkin, 1975), and other recent works (Attwell and Laughlin, 2001; Lennie, 2003) assume that the action potential requires four times $\mathrm{Na}^{+}$charge compared to the charge needed for the change in voltage. This waste of $\mathrm{Na}^{+}$charge, and accordingly metabolic energy, is the result of extensive overlap between 
inward $\mathrm{Na}^{+}$and outward $\mathrm{K}^{+}$during the generation of action potentials.

However, it has been demonstrated that mammalian central neurons, characterized by action potentials similar to those of the squid giant axon, are significantly more efficient in generating action potentials (Carter and Bean, 2009).

We show in this work that increased firing frequencies induced by higher temperatures in the Hodgkin and Huxley model imply more efficient use of sodium entry and metabolic energy. The paper is organized as follows. In section 2, the dynamics and electrochemical energy of the Hodgkin and Huxley model are introduced. In section 3 we discuss the overlap of ion currents and energy efficiency as a function of temperature in the squid axon. Finally, conclusions are drawn in section 4.

\section{MATERIALS AND METHODS}

\subsection{THE HODGKIN-HUXLEY NEURON ENERGY}

In the original Hodgkin-Huxley model (Hodgkin and Huxley, 1952), the dynamics governing the membrane potential is given by:

$$
\begin{aligned}
C \dot{V}= & -g_{N a} m^{3} h\left(V-E_{N a}\right) \\
& -g_{K} n^{4}\left(V-E_{K}\right)-g_{l}\left(V-E_{l}\right)+I,
\end{aligned}
$$

where $V$ is the membrane potential in $\mathrm{mV}, C$ the membrane capacitance density in $\mu \mathrm{F} / \mathrm{cm}^{2}, I$ is the total membrane current density in $\mu \mathrm{A} / \mathrm{cm}^{2} . g_{N_{a}}, g_{K}$, and $g_{l}$ are the maximal conductances per unit area for ion and leakage channels, and $E_{N a}, E_{K}$, and $E_{l}$ are the corresponding reversal potentials.

The gating variables $m, h$, and $n$, representing, respectively, sodium channels activation and deactivation variables, and potassium channels activation variable, obey the standard kinetic equation $\dot{x}=\alpha_{x}(1-x)-\beta_{x} x,(x=m, h, n)$, where $\alpha_{x}$ and $\beta_{x}$ are voltage-dependent variables. For sodium channels, the activation and deactivation rates are given by,

$$
\begin{aligned}
& \alpha_{m}(V)=(2.5-0.1 V) /(\exp (2.5-0.1 V)-1) \\
& \beta_{m}(V)=4 \exp (-V / 18) \\
& \alpha_{h}(V)=0.07 \exp (-V / 20) \\
& \beta_{h}(V)=1 /(\exp (3-0.1 V)+1)
\end{aligned}
$$

and for potassium channels,

$$
\begin{aligned}
& \alpha_{n}(V)=(0.1-0.01 V) /(\exp (1-0.1 V)-1), \\
& \beta_{n}(V)=0.125 \exp (-V / 80) .
\end{aligned}
$$

In this work we have used for these parameters the standard constant values given in Table 1 (Gerstner and Kistler, 2002).

The ion currents of sodium, potassium, and leakage (mainly chloride) correspond, respectively, to the three first terms in the right hand of the Equation (1), and are generated in response to a change in the respective ion conductances.

Figure 1 shows in part (A) the shape of the sodium and potassium currents corresponding to a particular action potential. The sodium current is negative but has been depicted with a positive sign for a better appreciation of the great extent of its overlapping
Table 1 | The parameters of the Hodgkin-Huxley equations.

\begin{tabular}{lcc}
\hline $\boldsymbol{x}$ & $\boldsymbol{g}_{\boldsymbol{x}}\left(\mathbf{m S} / \mathbf{c m}^{\mathbf{2}}\right)$ & $\boldsymbol{E}_{\boldsymbol{X}}(\mathbf{m V})$ \\
\hline$N_{a}$ & 120 & 115 \\
$\mathrm{~K}$ & 36 & -12 \\
$\mathrm{I}$ & 0.3 & 10.6 \\
\hline
\end{tabular}

The membrane capacitance density is $C=1 \mu \mathrm{F} / \mathrm{cm}^{2}$. The voltage scale is shifted so that the resting potential vanishes.

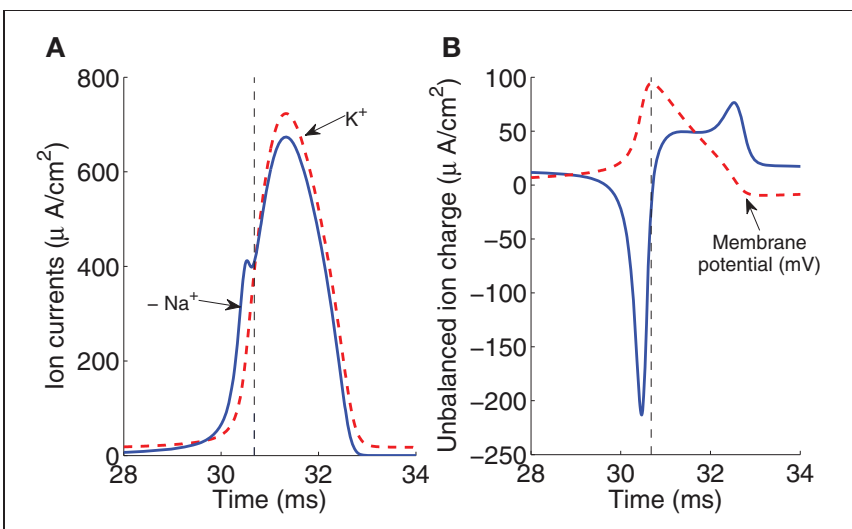

FIGURE 1 | (A) The currents of sodium and potassium ions during an action potential generated for an external stimulus $I=13 \mu \mathrm{A} / \mathrm{cm}^{2}$. (B) The unbalanced ion charge resulting from the overlapping and offsetting of $\mathrm{Na}^{+}$ and $\mathrm{K}^{+}$flux (the membrane potential is represented in dashed line). The rising and falling phases are separated by the vertical dashed line. The area under the curve in the rising phase region corresponds to the net unbalanced sodium ion charge crossing into the membrane during the rising of the action potential.

with the potassium current. Note that as sodium and potassium currents are both of positive charges but moving in opposite directions of the cell's membrane they neutralize each other to the extent of their mutual overlap. The sodium charge that is not counterbalanced by simultaneously flowing potassium charge is much smaller for a greater overlap.

The unbalanced current load, represented in Figure 1B, consists of two components which occur, respectively, during the depolarizing and hyperpolarizing phases of the membrane potential action. The integral of the first component of this unbalanced load gives the net $\mathrm{Na}^{+}$ion charge that is not counterbalanced by simultaneously flowing $\mathrm{K}^{+}$crossing into the membrane during the rising of the action potential. The integral of the total unbalanced ionic current is directly proportional to the number of ATP molecules required to restore the resting potential.

For the action potential represented in Figure 1B generated for an external stimulus $I=13 \mu \mathrm{A} / \mathrm{cm}^{2}$, the total sodium charge transfer computed as the integral of $\mathrm{Na}^{+}$current was $1168 \mathrm{nC} / \mathrm{cm}^{2}$ which agree with the estimate of $1098 \mathrm{nC} / \mathrm{cm}^{2}$ reported recently in Sengupta et al. (2010). Neutralized currents that account for the overlapping of Sodium and potassium fluxes give rise to an excessive overlap charge of about $1092 \mathrm{nC} / \mathrm{cm}^{2}$. This overlap has been calculated as the difference between the total $\mathrm{Na}^{+}$current and the depolarizing unbalanced component of $\mathrm{Na}^{+}$current. As stated in the work of Hodgkin, the squid action 
potential is very inefficient in the sense that it requires a fourfold $\mathrm{Na}^{+}$charge compared to the minimum charge necessary to depolarize a pure capacitor (Hodgkin, 1975). The efficiency of sodium entry during the generation of action potential in the squid axon at different temperatures is discussed in section 3 . The values of sodium and overlap load reported above correspond to a temperature of $6.3^{\circ} \mathrm{C}$.

To estimate the energy consumption necessary to restore the resting potential in the Hodgkin-Huxley model, we have used a new approach not based on the ion counting method. Following previous works of finding energy functions of neuron models of chaotic dynamics (Sarasola et al., 2004, 2005; Torrealdea et al., 2009), we have deduced for the model given by Equation (1) an energy function representing the analytical expression of the electrochemical energy involved in its dynamics. The procedure followed to find this energy has been reported in detail in Moujahid et al. (2011), and is summarized below.

It is well known that the Hodgkin-Huxley equation given by Equation (1) expresses an electrical circuit consisting of capacitor $C$ and three $\mathrm{Na}, \mathrm{K}$, and $\mathrm{L}$ ionic channels, where $g_{N_{a}}, g_{K}$, and $g_{l}$ are the maximal conductances, and batteries stand for the Nernst potentials of their corresponding ions. If $V$ is the membrane potential, the total electrical energy accumulated in the circuit at a given moment in time is,

$$
H(t)=\frac{1}{2} C V^{2}+H_{N_{a}}+H_{K}+H_{l}
$$

where the first term in the summation gives the electrical energy accumulated in the capacitor and represents the energy needed to create the membrane potential $V$ of the neuron. The other three terms are the respective energies in the batteries needed to create the concentration jumps in sodium, potassium, and chloride. The electrochemical energy accumulated in the batteries is unknown. Nevertheless, the rate of electrical energy provided to the circuit by a battery is known to be the electrical current through the battery times its electromotive force. Thus, the total derivative with respect to time of the above energy will be,

$$
\dot{H}(t)=C V \dot{V}+i_{N_{a}} E_{N_{a}}+i_{K} E_{K}+i_{l} E_{l}
$$

where $E_{N a}, E_{K}$, and $E_{l}$ are the Nernst potentials of the sodium, potassium and leakage ions in the resting state of the neuron. And $i_{N_{a}}, i_{K}$, and $i_{l}$ are the ion currents of sodium, potassium and leakage, given by,

$$
\begin{aligned}
i_{N_{a}} & =g_{N a} m^{3} h\left(V-E_{N a}\right), \\
i_{K} & =g_{K} n^{4}\left(V-E_{K}\right), \\
i_{l} & =g_{l}\left(V-E_{l}\right),
\end{aligned}
$$

If we substitute Equations (1) and (4) in Equation (3), we have for the energy rate in the circuit,

$$
\dot{H}=V I-g_{N_{a}} m^{3} h\left(V-E_{N_{a}}\right)^{2}-g_{K} n^{4}\left(V-E_{K}\right)^{2}-g_{l}\left(V-E_{l}\right)^{2},
$$

which provides the total derivative of the electrochemical energy in the neuron as a function of its state variables. The first term in the right hand summation represents the electrical power given to the neuron via the different junctions reaching the neuron and the other three terms of the summation represent the energy per second consumed by the ion channels. This equation permits evaluation of the total energy consumed by the neuron and also gives information about the consumption associated to each of the sodium, potassium, and leaking channels.

Figure 2A reports the time course of the electrochemical energy consumption in $\mathrm{nJ} / \mathrm{s}$ corresponding to sodium ion channel for the particular action potential (dashed line) generated at an external constant stimulus $I=13 \mu \mathrm{A} / \mathrm{cm}^{2}$. This energy consumption is given by the term $g_{N_{a}} m^{3} h\left(V-E_{N_{a}}\right)^{2}$ [see Equation (5)]. The total metabolic consumption given by the sum of the last three terms in Equation (5) is reported in Figure 2B. The last three terms of the energy derivative are negative definite, corresponding to an actual energy dissipation of energy, but has been represented as a positive consumption.

The total consumption of energy per second at the ion channels required to generate one particular action potential must be replenished by the ion pumps and metabolically supplied by hydrolysis of ATP molecules in order to maintain the neuron's activity. The higher demand of metabolic energy associated to the generation of spikes is clearly visible, for instance at $I=13 \mu \mathrm{A} / \mathrm{cm}^{2}$ which is the value used to generate the particular action potential analyzed previously, the average of the total metabolic consumption depicted in Figure 2B is about $11.4 \mu \mathrm{J} / \mathrm{s}$ per membrane unit area. This consumption must be replenished by metabolic ATP supply. The number of ATP molecules per membrane unit area hydrolyzed by the $\mathrm{Na}^{+} / \mathrm{K}^{+}$ATPase pump to extrude the $\mathrm{Na}^{+}$load can be deduced from the amount of $\mathrm{Na}^{+}$ ions crossing the membrane during an action potential, operating
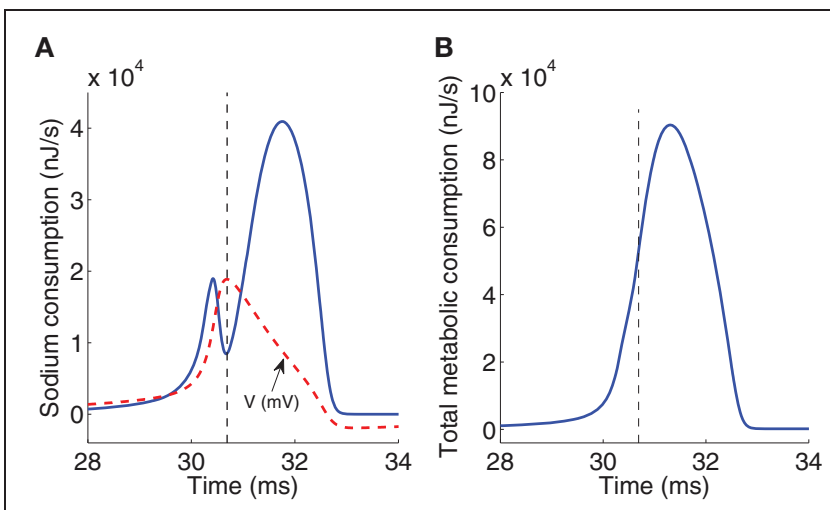

FIGURE 2 | (A) Electrochemical energy consumption corresponding to sodium ions at an action potential (dashed line) generated for an external stimulus $I=13 \mu \mathrm{A} / \mathrm{cm}^{2}$. Values of membrane potential are scaled with a factor of 200. (B) Total metabolic consumption corresponding to three ion channels at the same action potential. Magnitudes refer to $\mathrm{nJ} / \mathrm{s}$. Vertical dashed line in panels $(\mathbf{A})$ and $(\mathbf{B})$ separates between the rising and falling phase regions. 
with a ratio of $3 \mathrm{Na}^{+}$per ATP (Attwell and Laughlin, 2001; Crotty et al., 2006).

The ratio of the total metabolic consumption (in J/s) to one third of the number of $\mathrm{Na}^{+}$load through the membrane expressed in electronvolts per ATP represents the efficiency of the ATP hydrolysis measured as the free energy provide by the hydrolysis of one molecule of ATP. We will show that our calculation of the actual energy consumption and the number of ATP molecules involved in the generation of an action potential are consistent with relevant data in the literature and that the Hodgkin-Huxley model produces accurate estimates of energy consumption.

\section{RESULTS}

\subsection{ION CURRENTS OVERLAPPING}

The overlap of ion currents in the Hodgkin-Huxley model decreases as the temperature increases and the impact of overlap on the number of ATP molecules required per action potential can be analyzed rescaling the model equations to include the temperature-dependence. In this work, we have adopted the original assumption of Hodgkin and Huxley multiplying the rates of change of the activation $m, n$, and inactivation $h$ gating variables by a factor $k=3^{(T-6.3) / 10}, T\left[{ }^{\circ} \mathrm{C}\right]$ (Chandler and Meves, 1970).

To illustrate the overlap decrease with increasing temperature, Figure 3 shows instantaneous $\mathrm{Na}^{+}$and $\mathrm{K}^{+}$currents elicited by a single spike at, respectively, $6.3^{\circ} \mathrm{C}$ and $18.5^{\circ} \mathrm{C}$. To perform the simulation we have rescaled the current equations to different temperatures between $6.3^{\circ} \mathrm{C}$ and $18.5^{\circ} \mathrm{C}$ which is the range of temperatures in the original study of the squid giant axon by Hodgkin and Huxley. It should be noticed that for higher temperature the firing regime in the Hodgkin-Huxley model can only be maintained at large values of the injected current.

To quantify the current overlap we have adopted two different measures. Following (Attwell and Laughlin, 2001), we calculated the dimensionless charge separation as the $\mathrm{Na}^{+}$charge that is not counterbalanced by simultaneously flowing $\mathrm{K}^{+}$charge (depolarizing component of the unbalanced load depicted in Figure 1B divided by total $\mathrm{Na}^{+}$charge per action potential).
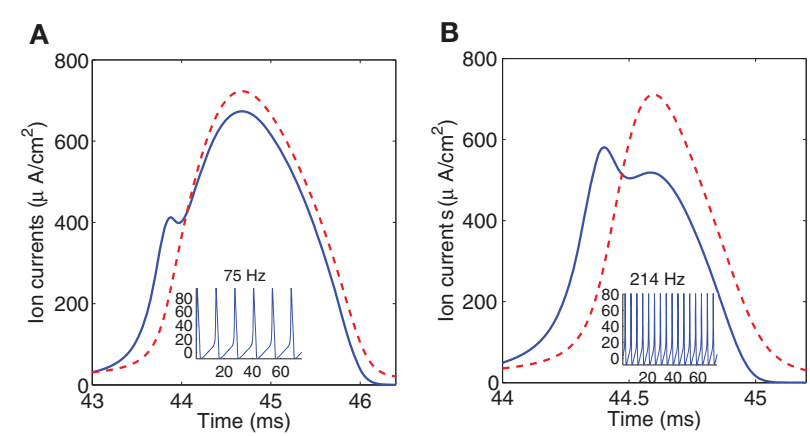

FIGURE 3 | Simulated sodium (solid line) and potassium (dashed line) currents at $6.3^{\circ} \mathrm{C}(\mathrm{A})$, and $18.5^{\circ} \mathrm{C}(\mathrm{B})$, showing different degree of

overlap. Firing frequencies are 75 and $214 \mathrm{~Hz}$, respectively. Sodium current is reversed for comparison.
The relationship between charge separation and temperature is illustrated in the inset of Figure 4A.

As it can be appreciated, charge separation shows a 2.98 -fold increase with increasing temperature and varies from 0.0652 at $6.3^{\circ} \mathrm{C}$ to 0.1942 at $18.5^{\circ} \mathrm{C}$. At this temperature, Alle et al. (2009) studying mossy fibers of the rat hippocampus report an average charge separation of 0.769 . The respective consumptions per action potential reflect this different overlap. At $18.5^{\circ} \mathrm{C}$ with an injection current $I=13 \mu \mathrm{A} / \mathrm{cm}^{2}$, the Hodgkin-Huxley model of the squid giant axon demands $0.68 \times 10^{12} \mathrm{ATP} / \mathrm{cm}^{2}$ to produce one action potential (see Table 2), while according to Alle et al. (2009), mossy fibers of the rat hippocampus demand only $0.32 \times 10^{12} \mathrm{ATP} / \mathrm{cm}^{2}$ per action potential.

The collected results for the number of ATP molecules per unit membrane area to produce one action potential related to charge separation are depicted in Figure 4A. As it can be seen the increase in separation implies a 3.54-fold decrease in the number of ATP molecule $/ \mathrm{cm}^{2}$. At $6.3^{\circ} \mathrm{C}$ the $\mathrm{Na}^{+}$charge transfer per unit membrane area of an action potential in the squid axon is about $1168 \mathrm{nC} / \mathrm{cm}^{2}$, consuming $2.4310^{12}$ ATP molecules $/ \mathrm{cm}^{2}$. While at $18^{\circ} \mathrm{C}$, the $\mathrm{Na}^{+}$load is $346 \mathrm{nC} / \mathrm{cm}^{2}$ consuming $0.7210^{12}$ ATP molecules $/ \mathrm{cm}^{2}$. So, high frequency firing induced by high temperature appears to be more efficient in the use of $\mathrm{Na}^{+}$entry. Table 2 reports details of values achieved for these measures at different values of temperature.

The other measure used in this work to quantify the current overlap has dimension of charge and is computed following (Crotty et al., 2006) as the difference between the total $\mathrm{Na}^{+}$ load and the depolarizing component of the $\mathrm{Na}^{+}$load. Figure 4B shows the relationship between the total $\mathrm{Na}^{+}$load and the overlap load measured in $\mathrm{nC} / \mathrm{cm}^{2}$. As it can be seen the total $\mathrm{Na}^{+}$charge increases linearly with overlap load with a slope close to unity. That is, the overlap load is positively correlated with the total $\mathrm{Na}^{+}$charge that crosses the membrane resulting in a decisive factor when analyzing the efficiency. The same behavior is observed when considering the relationship between the total unbalanced load computed as the sum of $\mathrm{Na}^{+}$and $\mathrm{K}^{+}$currents, and the overlap load.
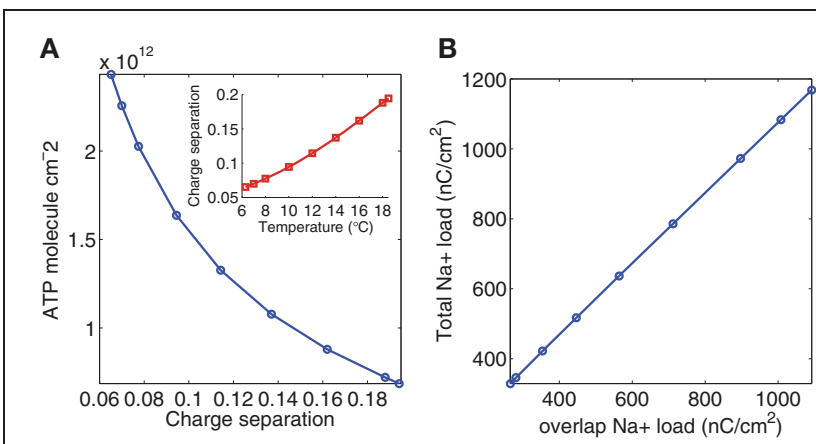

FIGURE 4 | (A) The number of ATP molecules required per action potential versus charge separation. In the inset charge separation measured as $\mathrm{Na}^{+}$ charge that is not counterbalanced by simultaneously flowing $\mathrm{K}^{+}$charge divided by total charge per action potential. (B) The relation between the total $\mathrm{Na}^{+}$load and the overlap charge, calculated as the difference between the total $\mathrm{Na}^{+}$current and its depolarizing component. 
Table 2 | Simulation values at different temperatures between $6.3^{\circ} \mathrm{C}$ and $18.5^{\circ} \mathrm{C}$ of the overlap between $\mathrm{N}^{+}$and $\mathrm{K}^{+}$ion currents in the squid giant axon.

\begin{tabular}{llllllll}
\hline Temperature $\left({ }^{\circ} \mathrm{C}\right)$ & 6.3 & 8 & 10 & 12 & 14 & 16 & 18 \\
Firing rate $(\mathrm{Hz})$ & 75 & 88 & 106 & 127 & 150 & 177 & 206 \\
Metabolic consumption $\left(\mathrm{n} / / \mathrm{cm}^{2}\right)$ & 152.3 & 126.9 & 102.6 & 83.2 & 67.7 & 55.3 & 45.4 \\
Total $\mathrm{Na}^{+}$load $\left(\mathrm{nC} / \mathrm{cm}^{2}\right)$ & 1168 & 973 & 786 & 637 & 518 & 422 & 346 \\
Overlap + load $\left(\mathrm{nC} / \mathrm{cm}^{2}\right)$ & 1092 & 897 & 712 & 564 & 447 & 354 & 281 \\
$\mathrm{Na}^{+}(\mathrm{pmole} / \mathrm{spike})$ & 12.12 & 10.09 & 8.15 & 6.6 & 5.37 & 4.38 & 3.58 \\
$\mathrm{ATP} \times 10^{12}$ (molecule/spike) & 2.43 & 2.02 & 1.63 & 1.32 & 1.07 & 0.87 & 0.72 \\
\hline
\end{tabular}

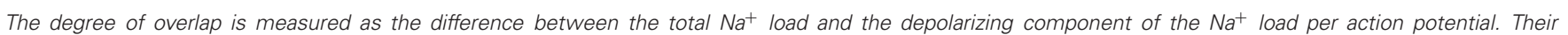

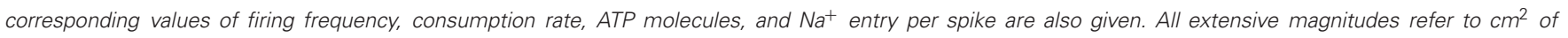
membrane.

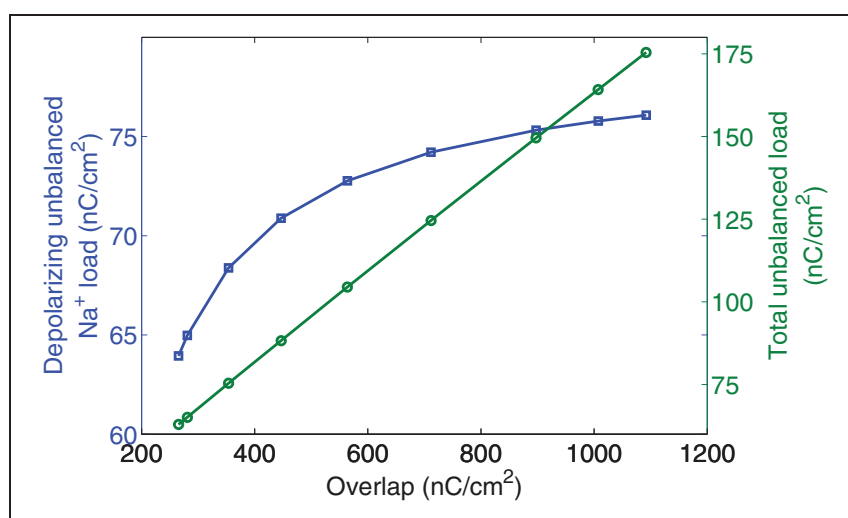

FIGURE 5 | Hodgkin-Huxley model scaled for different temperatures between $6.3^{\circ} \mathrm{C}$ and $18.5^{\circ} \mathrm{C}$. In square markers, the unbalanced $\mathrm{Na}^{+}$load crossing the membrane during the rising phase of the action potential as a function of overlap load. In circle markers, is represented the total unbalanced load calculated as the sum of sodium and potassium currents related to the overlap load.

Figure 5 reports, in the right axis, the collected values of the total unbalanced load related to the overlap load. And, in the left axis, the unbalanced component of $\mathrm{Na}^{+}$load crossing the membrane during the rising phase as a function of overlap. At $6.3^{\circ} \mathrm{C}$ the total unbalanced load is $175 \mathrm{nC} / \mathrm{cm}^{2}$ which is 2.3 times the depolarizing unbalanced $\mathrm{Na}^{+}$load, while at $18^{\circ} \mathrm{C}$ both measures provide close values around $65 \mathrm{nC} / \mathrm{cm}^{2}$. We observe that for a decrease of overlap load between its maximum value achieved at $6.3^{\circ} \mathrm{C}$ and values around $560 \mathrm{nC} / \mathrm{cm}^{2}$ corresponding to a temperature of about $12^{\circ} \mathrm{C}$, the depolarized unbalanced load undergoes only a slight decrease. However, further increase of temperature causes the overlap to decrease by 2.12-fold resulting in a 1.13-fold decrease of the depolarizing unbalanced $\mathrm{Na}^{+}$load.

\subsection{ENERGY EFFICIENCY}

The calculated values of the electrochemical energy involved in the dynamics of the Hodkin-Huxley model according to our method are reported in Figure 6. Both the total metabolic consumption and the metabolic consumption in the ionic channels show a decreasing pattern as the temperature increases. At $6.3^{\circ} \mathrm{C}$, the total action potential energy cost is around $152 \mathrm{~nJ} / \mathrm{cm}^{2}, 45 \%$
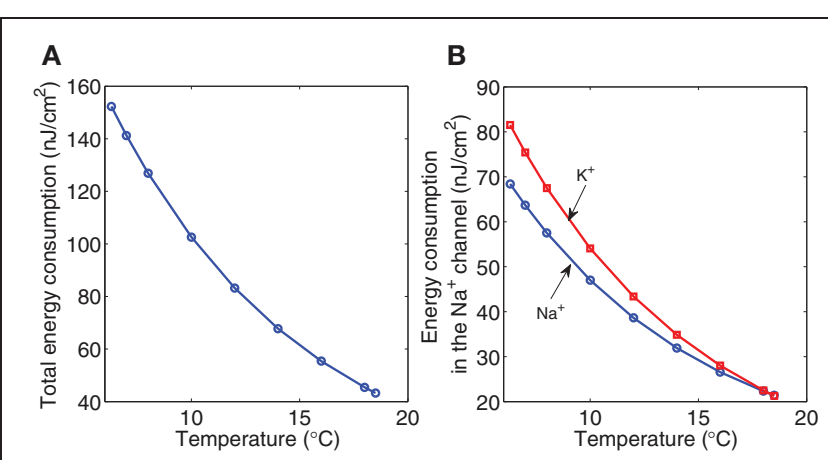

FIGURE 6 | The Hodgkin-Huxley model scaled for different temperatures between $6.3^{\circ} \mathbf{C}$ and $18.5^{\circ} \mathbf{C}$. (A) The total metabolic consumption required per action potential versus temperature. (B) The metabolic consumption in the $\mathrm{Na}^{+}$(circle marker) and $\mathrm{K}^{+}$(square marker) channels related to temperature. All magnitudes refer to $\mathrm{cm}^{2}$ of membrane.

of which is consumed in the sodium channel, and the rest is mainly consumed in the potassium channel. While at $18^{\circ} \mathrm{C}$ the energy consumed in both channels represents $49 \%$ of the total metabolic consumption. At this temperature the total metabolic consumption experiences a 3.35-fold significant decrease.

The total cost of one pump's cycle, that pumps three $\mathrm{Na}^{+}$ ions out of the cell and two $\mathrm{K}^{+}$ion in, is computed as the ratio of the total metabolic consumption [given by the last three terms of Equation (5)] to one third of the total number of $\mathrm{Na}^{+}$ load. According to our calculations, the liberated free energy by hydrolyzing one ATP molecule, defined as hydrolysis efficiency, seem to be independent of temperature and shows values around $0.39 \mathrm{eV}$ which is close to other estimates in the literature (Nelson, 2004; Sinkala, 2006).

However, if we consider only the depolarizing metabolic consumption in the $\mathrm{Na}^{+}$channel associated to the depolarizing unbalanced $\mathrm{Na}^{+}$load, the hydrolysis efficiency seem to be affected by temperature showing a parabolic shape with a minimum around $0.38 \mathrm{eV}$ (see Figure 7A). This minimum is observed for a temperature around $13^{\circ} \mathrm{C}$ corresponding to a firing frequency of about $138 \mathrm{~Hz}$.

The same behavior (see Figure 7B) was observed when studying the depolarizing efficiency as a function of the sodium 
channel conductance. The range of variation of the ion channel conductances was obtained multiplying the maximum conductances $g_{N a}, g_{K}$, and $g_{l}$ in the Hodgkin-Huxley model by the expression $k=1.5^{(T-6.3) / 10}$ for temperatures ranging between $6.3^{\circ} \mathrm{C}$ and $18.5^{\circ} \mathrm{C}$ according to Chandler and Meves (1970). The maximum depolarizing efficiency occurs for a sodium channel conductance value around $160 \mathrm{mS} / \mathrm{cm}^{2}$ which is close to the biological density conductance. Crotty et al. (2006), studying the energetic cost that arise from an action potential using computational models of the squid axon as a function of sodium channel densities, showed that the energy cost associated with the action potential produce a convex
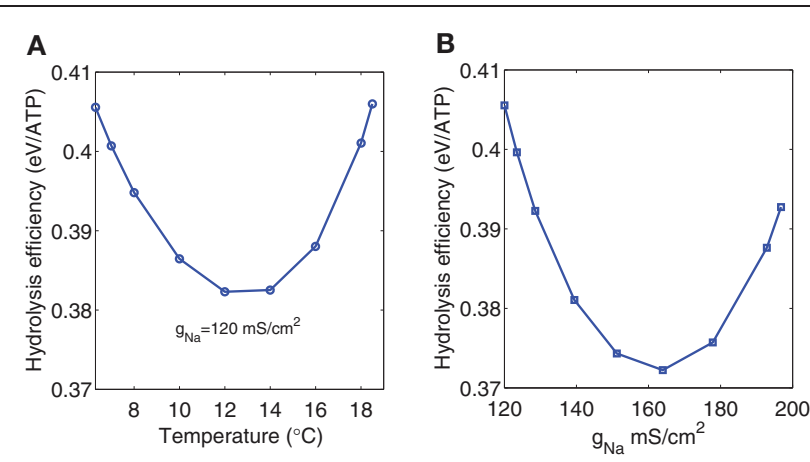

FIGURE 7 | (A) The ratio of the depolarizing $\mathrm{Na}^{+}$energy consumption to one third of the depolarizing unbalanced $\mathrm{Na}^{+}$load as a function of temperature. (B) The ratio of the depolarizing $\mathrm{Na}^{+}$energy consumption to one third of the depolarizing unbalanced $\mathrm{Na}^{+}$load as a function of sodium channel conductance. The range of variation of the $\mathrm{Na}^{+}$channel conductance was obtained multiplying the maximum conductances in the Hodgkin-Huxley model by the factor $k=1.5^{(T-6.3) / 10}$ for temperatures ranging between $6.3^{\circ} \mathrm{C}$ and $18.5^{\circ} \mathrm{C}$. curve with a minimum around the same value reported above.

We have seen that the fast-spiking regime in the HodgkinHuxley model induced by higher temperatures implies more efficient use of sodium entry, and gives rise to energy efficient action potentials characterized by less overlap between sodium and potassium currents. Since this firing regime could be also induced by raising the external stimulus, it would be interesting to investigate whether the same fast-spiking regime achieved, for one hand, by raising the temperature, and for the other hand, by increasing the external current, contributes in the same way in reducing both the energy consumption and the overlap load. To do so, we have computed the energy consumption required to generate an action potential and the corresponding firing frequency for different values of the external stimulus $\mathrm{I}$. We have considered values of I ranging between $13 \mu \mathrm{A} / \mathrm{cm}^{2}$ and $40 \mu \mathrm{A} / \mathrm{cm}^{2}$.

As it can be appreciated in Figure 8 (left panel), the influence of higher stimulus on the firing frequency in the squid giant axon is more significant only for higher temperatures. In fact, at a given temperature, raising the external current causes the firing frequency to increase by 1.46-fold, while at a given external stimulus, the firing frequency experiences a 2.96-fold increase with increasing temperature. On the other hand, according to our results, the fast-spiking regime induced by higher stimuli is less efficient in generating action potentials expending more energy compared with the same fast-spiking regime induced by higher temperatures. For example, at $T=8^{\circ} \mathrm{C}$ and a relatively high external current $I=39 \mu \mathrm{A} / \mathrm{cm}^{2}$ (see circle markers in Figure 8), the firing frequency of the squid axon is of about $127 \mathrm{~Hz}$, and are necessary $106.75 \mathrm{~nJ} / \mathrm{cm}^{2}$ to generate one action potential. While at a higher temperature $T=12^{\circ} \mathrm{C}$ and a low current $I=13 \mu \mathrm{A} / \mathrm{cm}^{2}$ (see square markers in Figure 8) corresponding to the same firing regime (i.e., $F=127 \mathrm{~Hz}$ ), the energy consumption is only

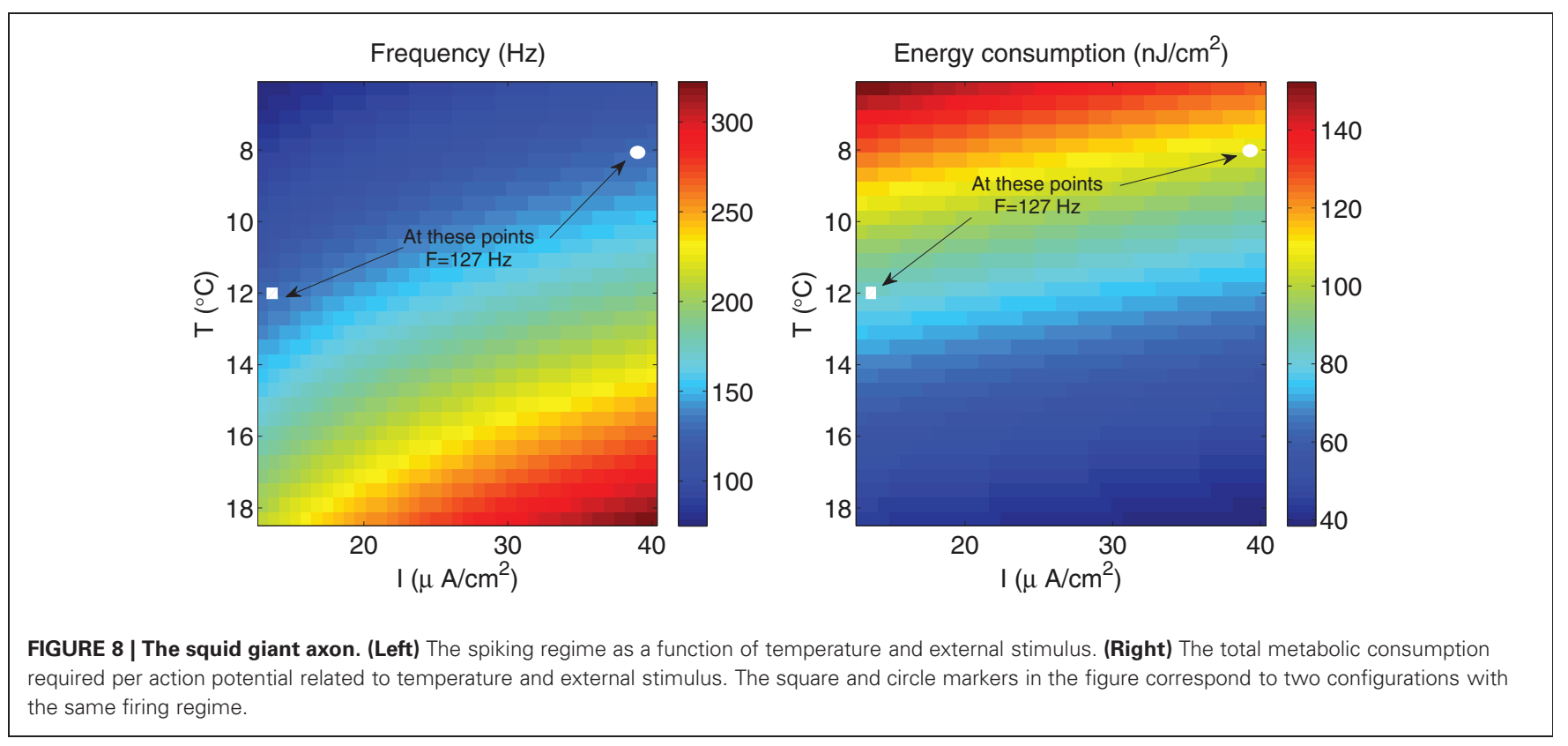


$83.24 \mathrm{~nJ} / \mathrm{cm}^{2}$. This 0.78 -fold decrease in energy consumption is accompanied by a 0.76 -fold decrease in overlap load between the spike-generating $\mathrm{Na}^{+}$current and delayed rectifier $\mathrm{K}^{+}$current. The corresponding values of overlap load are $740.83 \mathrm{nC} / \mathrm{cm}^{2}$ and $563.92 \mathrm{nC} / \mathrm{cm}^{2}$, respectively. This confirms again that the overlap load of voltage-gated currents of $\mathrm{Na}^{+}$and $\mathrm{K}^{+}$dominates energy efficiency, and efficient action potentials have little overlap.

\section{DISCUSSIONS}

Our results with simulated spikes of the squid axon show that increased firing frequencies induced by higher temperatures imply more efficient use of sodium entry due mainly to the reduced overlap load between inward $\mathrm{Na}^{+}$current and outward $\mathrm{K}^{+}$current. This, corroborates what has been reported recently in Carter and Bean (2009); Sengupta et al. (2010), and Alle et al. (2009), i.e., the most energy efficient action potentials are those generated by $\mathrm{Na}^{+}$and $\mathrm{K}^{+}$currents that have substantially reduced overlap.

The values of sodium entry are close to the original values calculated by Hodgkin and Huxley (1952), and are in nice agreement with values reported recently by Sengupta et al. (2010). At $6.3^{\circ} \mathrm{C}$ corresponding to $75 \mathrm{~Hz}$ we obtain a sodium influx of $12.12 \mathrm{pmole} / \mathrm{cm}^{2}$ per spike $\left(1168 \mathrm{nC} / \mathrm{cm}^{2}\right)$ while at $18^{\circ} \mathrm{C}$ and $206 \mathrm{~Hz}$ the sodium influx is $3.58 \mathrm{pmole} / \mathrm{cm}^{2}$ per spike $\left(346 \mathrm{nC} / \mathrm{cm}^{2}\right)$ which means a 3.38 -fold decrease in sodium entry corresponding to a 2.75 -fold increase in firing frequency.

Regarding the energy consumption associated to the generation of action potentials in the squid axon, we have found that the hydrolysis of one ATP molecule liberates a free energy with optimum values that range from $0.37 \mathrm{eV}$ achieved for a sodium conductance around $160 \mathrm{mS} / \mathrm{cm}^{2}$ and when only the depolarizing components of both energy consumption and $\mathrm{Na}^{+}$load are considered. To a value of about $0.39 \mathrm{eV}$ produced when considering the total metabolic consumption associated to the total $\mathrm{Na}^{+}$load.
As stated before, these values of hydrolysis efficiency are in nice agreement with other estimates, which confirms that our method of calculation of the actual energy consumption by the pump and the number of ATP molecules involved are consistent with other data in the literature.

Also, we have found that the fast-spiking regime in the Hodgkin-Huxley model induced by a higher stimulus appears to be less efficient in generating action potentials expending more energy compared with the same fast-spiking regime induced by a higher temperature. Accordingly, the reduction in the overlap of the $\mathrm{Na}^{+}$and $\mathrm{K}^{+}$currents is less when the firing frequency is raised by rising the external stimulus. Carter and Bean (2009), have suggested that the primary determinant of differences in $\mathrm{Na}^{+}$ entry efficiency among neurons is their different action potential shapes. Indeed, the shape of the action potential in the squid axon at a given frequency is different depending on whether it has been generated by raising the temperature or the external stimulus.

The findings of this work were validated using others Hodgkin-Huxley-like model neurons, in particular, we have considered the simplest model of regular spiking cells in neocortex which consists of sodium and potassium currents responsible for generating spikes, and an additional slow voltage-dependent potassium current responsible for spike-frequency adaptation. This model generates action potentials which capture the typical firing characteristics of regular spiking neurons in ferret visual cortex in vivo (Pospischil et al., 2008). To carry out the comparison, we performed the same experiment as for the squid giant axon, i.e., rescaling the model equations given in Pospischil et al. (2008) to include the temperature-dependence of the membrane ionic conductances, using values that range between $20^{\circ} \mathrm{C}$ and $40^{\circ} \mathrm{C}$ which corresponds to the normal range of temperature in these cells. The external current was varied between 1.5 and $5 \mu \mathrm{A} / \mathrm{cm}^{2}$. The results (see Figure 9) show a behavior qualitatively
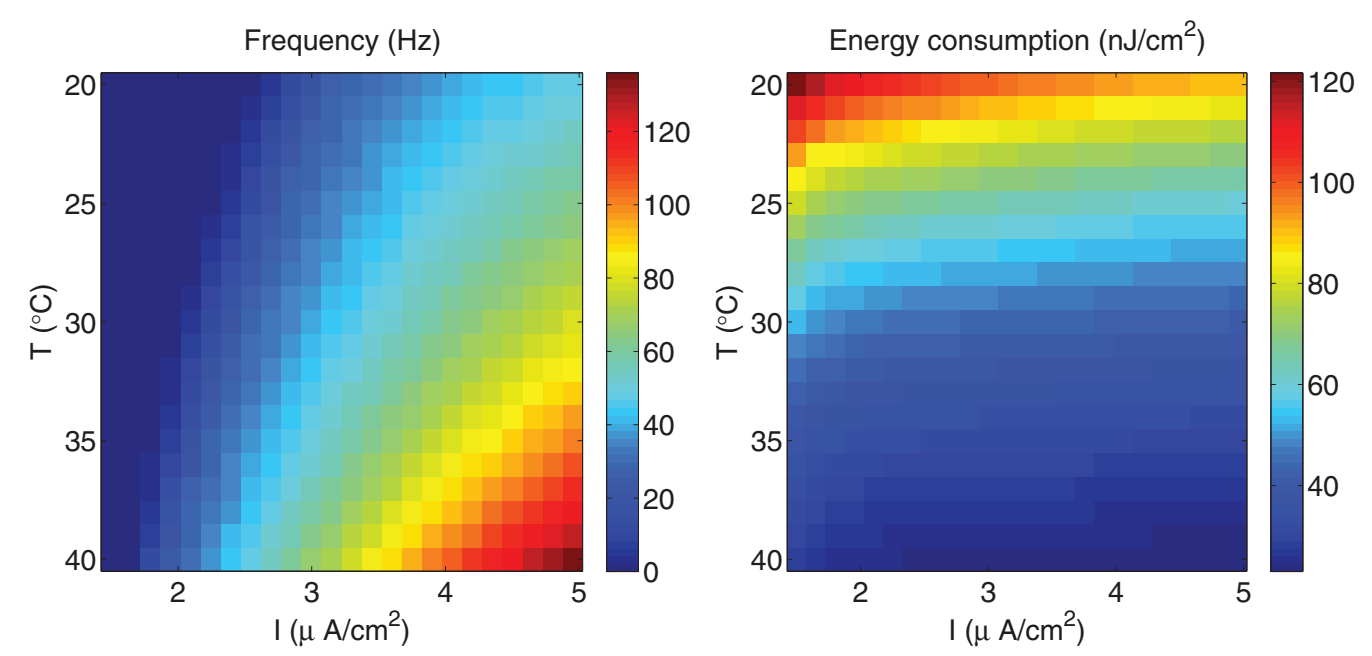

FIGURE 9 | The regular-spiking model reproducing the typical firing characteristics of regular spiking neurons in ferret visual cortex in vivo. (Left) The firing regime as a function of temperature and external stimulus. (Right) The total metabolic consumption required per action potential related to temperature and external stimulus. 
similar to that we observed in the squid axon. i.e., less energy is spent per action potential at higher temperatures than at lower ones due mainly to the reduced overlap between sodium and potassium currents. And, the efficiency of action potentials is more dependent on temperature than on the external stimulus. At $T=20^{\circ} \mathrm{C}$ and an external high stimulus of about $4.75 \mu \mathrm{A} / \mathrm{cm}^{2}$, the regular spiking cell fires at a frequency of about $46.5 \mathrm{~Hz}$, and requires $90.5 \mathrm{~nJ} / \mathrm{cm}^{2}$ to generate one action potential. While, for a higher temperature $T=36.5^{\circ} \mathrm{C}$ and relatively a small current $I=2.75 \mu \mathrm{A} / \mathrm{cm}^{2}$, making the cell to fire at the same firing regime (i.e., frequency of about $46.5 \mathrm{~Hz}$ ), the energy consumption is $29 \mathrm{~nJ} / \mathrm{cm}^{2}$ representing only one third of the energy expended when considering higher stimulus. This difference in energy

\section{REFERENCES}

Alle, H., Roth, A., and Geiger, J. R. P. (2009). Energy-efficient action potentials in hippocampal mossy fibers. Science 325, 1405-1408.

Ames III, A. (2000). CNS energy metabolism as related to function. Brain Res. Rev. 34, 42-68.

Attwell, D., and Laughlin, S. B. (2001). An energy budget for signaling in the grey matter of the brain. J. Cereb. Blood Flow Metab. 21, 1133-1145.

Belanger, M., Allaman, I., and Magistretti, P. J. (2011). Brain energy metabolism: focus on astrocyte-neuron metabolic cooperation. Cell Metab. 14, 724-738.

Carter, B. C., and Bean, B. P. (2009). Sodium entry during action potentials of mammalian neurons: incomplete inactivation and reduced metabolic efficiency in fast-spiking neurons. Neuron 64, 898-909.

Chandler, W. K., and Meves, H. (1970). Rate constants associated with changes in sodium conductance in axons perfused with sodium fluoride. J. Physiol. 211, 679-705.

Crotty, P., Sangrey, T., and Levy, W. B. (2006). Metabolic energy cost of action potential velocity. $J$. Neurophysiol. 96, 1237-1246.

Falk, D. (1990). Brain evolution in Homo: the radiator theory. Behav. Brain Sci. 13, 333-381.
Gerstner, W., and Kistler, W. (2002). Spiking Neuron Models: Single Neurons, Populations, Plasticity. Cambridge, UK: Cambridge University Press.

Hodgkin, A. L. (1975). The optimum density of sodium channels in an unmyelinated nerve. Philos. Trans. $R$ Soc B Biol. Sci. 270, 297-300.

Hodgkin, A. L., and Huxley, A. F. (1952). A quantitative description of membrane current and its application to conduction and excitation in nerve. J. Physiol. 117, 500-544.

Kiyatkin, E. A. (2007). Brain temperature fluctuations during physiological and pathological conditions. Eur. J. Appl. Physiol. 101, 3-17.

Koch, C. (1998). Biophysics of Computation: Information Processing in Single Neurons. New York, NY: Oxford University Press.

Laughlin, S. B. (2001). Energy as a constraint on the coding and processing of sensory information. Curr. Opin. Neurobiol. 11, 475-480.

Laughlin, S. B., de Ruyter van Steveninck, R. R., and Anderson, J. C. (1998). The metabolic cost of neural information. Nat. Neurosci. 1, 36-41.

Lennie, P. (2003). The cost of cortical computation. Curr. Biol. 13, 493-497.

Moujahid, A., d'Anjou, A., Torrealdea, F. J., and Torrealdea, F. (2011). Energy and information in

consumption is due to a significant reduction of the overlap load that decreases from 506 to $88 \mathrm{nC} / \mathrm{cm}^{2}$.

Our principal findings were that the energy consumption required to generate action potentials in the squid giant axon as well as in the regular-spiking model of cells in neocortex is lower at higher temperatures. Also, we found that for these cells, the fast-spiking regimes induced by higher temperatures are more energy efficient than those induced by higher stimuli. Finally, we think that the approach considered in this work could bring a new framework to analyze the relationship between energy consumption, temperature, and firing frequency in neuronal tissues since it could be potentially used for more relevant models of mammalian brains.

Hodgkin-Huxley neurons. Phys. Rev. E 83, 031912.

Nelson, P. (2004). Biological Physics: Energy, Information, Life. New York/Basingstoke: W. H. Freeman and Company.

Pospischil, M., Toledo-Rodriguez, M. Monier, C., Piwkowska, Z., Bal, T. Frégnac, Y., et al. (2008). Minimal Hodgkin-Huxley type models for different classes of cortical and thalamic neurons. Biol. Cybern. 99, 427-441.

Sarasola, C., d'Anjou, A., Torrealdea, F. J., and Moujahid, A. (2005). Energy-like functions for some dissipative chaotic systems. Int. J. Bifurcat. Chaos Appl. Sci. Eng. 15, 2507-2521.

Sarasola, C., Torrealdea, F. J., d'Anjou, A., Moujahid, A., and Grana, M. (2004). Energy balance in feedback synchronization of chaotic systems. Phys. Rev. E 69, 011606.

Sengupta, B., Stemmler, M., Laughlin, S. B., and Niven, J. E. (2010). Action potential energy efficiency varies among neuron types in vertebrates and invertebrates. PLoS Comput. Biol. 6:e1000840. doi:10.1371/journal.pcbi.1000840

Shulman, R. G., Rothman, D. L., Behar, K. L., and Hyder, F. (2004). Energetic basis of brain activity: implications for neuroimaging. Trends Neurosci. 27, 489-495.
Siekevitz, P. (2004). Producing neuronal energy. Science 306, 410-411.

Sinkala, Z. (2006). Soliton/exciton transport in proteins. J. Theor. Biol. 241, 919-927.

Torrealdea, F. J., Sarasola, C., d'Anjou, A., Moujahid, A., and de Mendizabal, N. V. (2009). Energy efficiency of information transmission by electrically coupled neurons. Biosystems 97, 60-71.

Conflict of Interest Statement: The authors declare that the research was conducted in the absence of any commercial or financial relationships that could be construed as a potential conflict of interest.

Received: 03 September 2012; accepted: 29 October 2012; published online: 15 November 2012.

Citation: Moujahid $A$ and d'Anjou A (2012) Metabolic efficiency with fast spiking in the squid axon. Front. Comput. Neurosci. 6:95. doi: 10.3389/ fncom.2012.00095

Copyright (C) 2012 Moujahid and d'Anjou. This is an open-access article distributed under the terms of the Creative Commons Attribution License, which permits use, distribution and reproduction in other forums, provided the original authors and source are credited and subject to any copyright notices concerning any third-party graphics etc. 